\title{
The hybrid power mean of the quartic Gauss sums and the two-term exponential sums
}

\section{Xiaoxue $\mathrm{Li}^{1^{*}}$}

\section{"Correspondence:}

Ixx20072012@163.com

${ }^{1}$ Faculty of Science, Xi'an

Aeronautical University, Xi'an,

P.R. China

\section{Springer}

\begin{abstract}
In this paper, we use the analytic method and the properties of classical Gauss sums to study the computational problems of one kind hybrid power mean of quartic Gauss sums and two-term exponential sums, and give an interesting fourth-order linear recurrence formula for it.

MSC: 11L05; 11L07

Keywords: Quartic Gauss sums; Two-term exponential sums; Hybrid power mean; Analytic method
\end{abstract}

\section{Introduction}

Let $q \geq 3$ be an integer. For any positive integer $k \geq 2$, the $k$ th Gauss sums $G(m, k ; q)$ are defined as

$$
G(m, k ; q)=\sum_{a=0}^{q-1} e\left(\frac{m a^{k}}{q}\right),
$$

where, as usual, $e(y)=e^{2 \pi i y}$.

Recently, some scholars have studied the properties of $G(m, 4 ; p)$ and obtained many interesting results, where $p$ is an odd prime with $p \equiv 1 \bmod 4$. For example, Shimeng Shen and Wenpeng Zhang [1] proved a recurrence formula related to $G(m, 4 ; p)$. The author and Jiayuan $\mathrm{Hu}[2]$ studied the computational problem of the hybrid power mean

$$
\sum_{b=1}^{p-1}\left|\sum_{a=0}^{p-1} e\left(\frac{b a^{4}}{p}\right)\right|^{2} \cdot\left|\sum_{c=1}^{p-1} e\left(\frac{b c+\bar{c}}{p}\right)\right|^{2} .
$$

We proved the identity

$$
\begin{aligned}
& \sum_{b=1}^{p-1}\left|\sum_{a=0}^{p-1} e\left(\frac{b a^{4}}{p}\right)\right|^{2} \cdot\left|\sum_{c=1}^{p-1} e\left(\frac{b c+\bar{c}}{p}\right)\right|^{2} \\
& \quad= \begin{cases}3 p^{3}-3 p^{2}-3 p+p\left(\tau^{2}\left(\bar{\chi}_{4}\right)+\tau^{2}\left(\chi_{4}\right)\right), & \text { if } p \equiv 5 \bmod 8 ; \\
3 p^{3}-3 p^{2}-3 p-p \tau^{2}\left(\bar{\chi}_{4}\right)-p \tau^{2}\left(\chi_{4}\right)+2 \tau^{5}\left(\bar{\chi}_{4}\right)+2 \tau^{5}\left(\chi_{4}\right), & \text { if } p \equiv 1 \bmod 8,\end{cases}
\end{aligned}
$$

(c) The Author(s) 2018. This article is distributed under the terms of the Creative Commons Attribution 4.0 International License (http://creativecommons.org/licenses/by/4.0/), which permits unrestricted use, distribution, and reproduction in any medium, provided you give appropriate credit to the original author(s) and the source, provide a link to the Creative Commons license, and indicate if changes were made. 
where $\chi_{4}$ denotes any fourth-order character $\bmod p, \tau(\chi)=\sum_{a=1}^{p-1} \chi(a) e\left(\frac{a}{p}\right)$ denotes the classical Gauss sums, and $\bar{c}$ denotes the multiplicative inverse of $c \bmod p$.

At the same time, the author and Jiayuan $\mathrm{Hu}$ [2] also pointed out how to compute the exact value of $\tau^{2}\left(\bar{\chi}_{4}\right)+\tau^{2}\left(\chi_{4}\right)$ and $\tau^{5}\left(\bar{\chi}_{4}\right)+\tau^{5}\left(\chi_{4}\right)$, these are two meaningful problems.

Zhuoyu Chen and Wenpeng Zhang [3] studied the properties of the Gauss sums

$$
G(k, p)=\tau^{k}(\psi)+\tau^{k}(\bar{\psi})
$$

By using the analytic method and the properties of classical Gauss sums, they obtained an exact computational formula for $G(k, p)$, which completely solved the problem proposed by the author and Jiayuan $\mathrm{Hu}$ in [2]. Some related works can also be found in references [4-11].

Inspired by reference [3], we will consider the following hybrid power mean:

$$
M_{k}(p)=\sum_{m=1}^{p-1}\left(\sum_{a=0}^{p-1} e\left(\frac{m a^{4}}{p}\right)\right)^{k} \cdot\left|\sum_{a=0}^{p-1} e\left(\frac{m a^{4}+a}{p}\right)\right|^{2} .
$$

For convenience, hereinafter, we always assume that $p$ is a prime with $p \equiv 1 \bmod 4,\left(\frac{*}{p}\right)=$ $\chi_{2}$ denotes the Legendre symbol $\bmod p$, and

$$
\alpha=\alpha(p)=\sum_{a=1}^{\frac{p-1}{2}}\left(\frac{a+\bar{a}}{p}\right)
$$

$\bar{a}$ denotes the solution of the equation $a x \equiv 1 \bmod p$. The number $\alpha$ is closely related to prime $p$. In fact, we have a very important formula

$$
p=\left(\sum_{a=1}^{\frac{p-1}{2}}\left(\frac{a+\bar{a}}{p}\right)\right)^{2}+\left(\sum_{a=1}^{\frac{p-1}{2}}\left(\frac{r a+\bar{a}}{p}\right)\right)^{2} \equiv \alpha^{2}+\beta^{2},
$$

where $r$ is any integer with $\left(\frac{r}{p}\right)=-1$ (see Theorems 4-11 in [12]).

In this paper, by using the analytic method, the properties of the classical Gauss sums, and trigonometric sums, we will study the computational problem of $M_{k}(p)$, and give an interesting fourth-order linear recurrence formula for it. That is, we will prove the following two results.

Theorem 1 If $p$ is a prime with $p \equiv 5 \bmod 8$, then for any integer $k \geq 4$, we have the linear recurrence formula

$$
M_{k}(p)=-2 p M_{k-2}(p)+8 p \alpha M_{k-3}(p)-p\left(9 p-4 \alpha^{2}\right) M_{k-4}(p)
$$

where the first four items in the sequence $\left\{M_{k}(p)\right\}$ are: $M_{0}(p)=p(p-3) ; M_{1}(p)=2 p \alpha$; $M_{2}(p)=-p\left(p^{2}-3 p-4 \alpha^{2}\right)$, and $M_{3}(p)=2 p^{2} \alpha(3 p-14)$.

Theorem 2 If $p$ is a prime with $p \equiv 1 \bmod 8$, then for any integer $k \geq 4$, we have the linear recurrence formula

$$
M_{k}(p)=6 p M_{k-2}(p)+8 p \alpha M_{k-3}(p)-p\left(p-4 \alpha^{2}\right) M_{k-4}(p),
$$


where the first four items in the sequence $\left\{M_{k}(p)\right\}$ are: $M_{0}(p)=p(p-3) ; M_{1}(p)=-6 p \alpha$; $M_{2}(p)=p\left(3 p^{2}-17 p-4 \alpha^{2}\right)$, and $M_{3}(p)=6 p^{2} \alpha(p-8)$.

For some special integers $k=2$ or $k=4$, from our theorems we may immediately deduce the following three corollaries.

Corollary 1 If $p$ is an odd prime with $p \equiv 5 \bmod 8$, then we have

$$
\sum_{m=1}^{p-1}\left|\sum_{a=0}^{p-1} e\left(\frac{m a^{4}}{p}\right)\right|^{2} \cdot\left|\sum_{c=0}^{p-1} e\left(\frac{m c^{4}+c}{p}\right)\right|^{2}=p\left(3 p^{2}-9 p-4 \alpha^{2}\right) .
$$

Corollary 2 If $p$ is an odd prime with $p \equiv 1 \bmod 8$, then we have the identity

$$
\sum_{m=1}^{p-1}\left|\sum_{a=0}^{p-1} e\left(\frac{m a^{4}}{p}\right)\right|^{2} \cdot\left|\sum_{c=0}^{p-1} e\left(\frac{m c^{4}+c}{p}\right)\right|^{2}=p\left(3 p^{2}-17 p-4 \alpha^{2}\right) .
$$

Corollary 3 If $p$ is an odd prime with $p \equiv 1 \bmod 8$, then we have

$$
\sum_{m=1}^{p-1}\left|\sum_{a=0}^{p-1} e\left(\frac{m a^{4}}{p}\right)\right|^{4} \cdot\left|\sum_{c=0}^{p-1} e\left(\frac{m c^{4}+c}{p}\right)\right|^{2}=p^{2}\left(17 p^{2}+4 p \alpha^{2}-99 p-84 \alpha^{2}\right) .
$$

Notes If $p=4 k+3$, then $\left(\frac{-1}{p}\right)=-1$. Then, in this case, for any integer $m$ with $(m, p)=1$, we have

$$
\begin{aligned}
\sum_{a=0}^{p-1} e\left(\frac{m a^{4}}{p}\right) & =1+\sum_{a=1}^{p-1}\left(1+\left(\frac{a}{p}\right)\right) e\left(\frac{m a^{2}}{p}\right) \\
& =\sum_{a=0}^{p-1} e\left(\frac{m a^{2}}{p}\right)+\sum_{a=1}^{p-1}\left(\frac{a}{p}\right) e\left(\frac{m a^{2}}{p}\right)=\sum_{a=0}^{p-1} e\left(\frac{m a^{2}}{p}\right)=\left(\frac{m}{p}\right) i \sqrt{p},
\end{aligned}
$$

where $i^{2}=-1$. Therefore, the hybrid power mean $M_{k}(p)$ can be easily obtained.

\section{Several lemmas}

To prove our main results, we need several simple lemmas. Here, we will use many properties of the classical Gauss sums, all of them can be found in reference [13], so they will not be repeated here. First we have the following lemma.

Lemma 1 If $p$ is a prime with $p \equiv 1 \bmod 4$, then for any fourth-order character $\psi \bmod p$, we have the identity

$$
\sum_{m=1}^{p-1} \psi(m)\left|\sum_{a=0}^{p-1} e\left(\frac{m a^{4}+a}{p}\right)\right|^{2}=-(1+\bar{\psi}(-1)) \sqrt{p} \tau(\bar{\psi})
$$

Proof First, from the trigonometric identity

$$
\sum_{m=1}^{q} e\left(\frac{n m}{q}\right)= \begin{cases}q & \text { if } q \mid n, \\ 0 & \text { if } q \nmid n,\end{cases}
$$


the properties of character $\psi \bmod p$ and noting that $\psi^{4}=\chi_{0}$, the principal character $\bmod p$, we have

$$
\begin{aligned}
\sum_{m=1}^{p-1} \psi(m)\left(\sum_{a=1}^{p-1} e\left(\frac{m a^{4}+a}{p}\right)\right) & =\sum_{a=1}^{p-1} e\left(\frac{a}{p}\right) \sum_{m=1}^{p-1} \psi(m) e\left(\frac{m a^{4}}{p}\right) \\
& =\tau(\psi) \sum_{a=1}^{p-1} \bar{\psi}\left(a^{4}\right) e\left(\frac{a}{p}\right)=\tau(\psi) \sum_{a=1}^{p-1} e\left(\frac{a}{p}\right)=-\tau(\psi) .
\end{aligned}
$$

Similarly, we also have

$$
\sum_{m=1}^{p-1} \psi(m)\left(\sum_{a=1}^{p-1} e\left(\frac{-m a^{4}-a}{p}\right)\right)=-\bar{\psi}(-1) \tau(\psi) .
$$

Since $p \equiv 1 \bmod 4$, so from the properties of the fourth-order character $\psi \bmod p$, we have

$$
\begin{aligned}
\sum_{m=1}^{p-1} \psi(m)\left|\sum_{a=1}^{p-1} e\left(\frac{m a^{4}+a}{p}\right)\right|^{2} & =\sum_{a=1}^{p-1} \sum_{b=1}^{p-1} \sum_{m=1}^{p-1} \psi(m) e\left(\frac{m b^{4}\left(a^{4}-1\right)+b(a-1)}{p}\right) \\
& =\tau(\psi) \sum_{a=1}^{p-1} \bar{\psi}\left(a^{4}-1\right) \sum_{b=1}^{p-1} e\left(\frac{b(a-1)}{p}\right) \\
& =-\tau(\psi) \sum_{a=1}^{p-1} \bar{\psi}\left(a^{4}-1\right) \\
= & -\tau(\psi) \sum_{a=1}^{p-1} \bar{\psi}(a-1)\left(1+\psi(a)+\psi^{2}(a)+\bar{\psi}(a)\right) \\
= & \bar{\psi}(-1) \tau(\psi)+\tau(\psi) \\
& -\tau(\psi) \sum_{a=1}^{p-1} \chi_{2}(a) \bar{\psi}(a-1)-\tau(\psi) \sum_{a=1}^{p-1} \bar{\psi}(a(a-1)),
\end{aligned}
$$

where $\psi^{2}=\chi_{2}=\left(\frac{*}{p}\right)$ denotes the Legendre symbol $\bmod p$.

From the properties of the classical Gauss sums, we have

$$
\begin{aligned}
\sum_{a=1}^{p-1} \chi_{2}(a) \bar{\psi}(a-1) & =\frac{1}{\tau(\psi)} \sum_{b=1}^{p-1} \psi(b) \sum_{a=1}^{p-1} \chi_{2}(a) e\left(\frac{b(a-1)}{p}\right) \\
& =\frac{\tau\left(\chi_{2}\right)}{\tau(\psi)} \sum_{b=1}^{p-1} \psi(b) \chi_{2}(b) e\left(\frac{-b}{p}\right)=\frac{\bar{\psi}(-1) \tau\left(\chi_{2}\right) \tau(\bar{\psi})}{\tau(\psi)} ; \\
\sum_{a=1}^{p-1} \bar{\psi}(a(a-1)) & =\frac{1}{\tau(\psi)} \sum_{b=1}^{p-1} \psi(b) \sum_{a=1}^{p-1} \bar{\psi}(a) e\left(\frac{b(a-1)}{p}\right) \\
& =\frac{\tau(\bar{\psi})}{\tau(\psi)} \sum_{b=1}^{p-1} \psi^{2}(b) e\left(\frac{-b}{p}\right)=\frac{\tau\left(\chi_{2}\right) \tau(\bar{\psi})}{\tau(\psi)} .
\end{aligned}
$$


Note that $\tau\left(\chi_{2}\right)=\sqrt{p}$, from (5), (6), and (7) we have

$$
\sum_{m=1}^{p-1} \psi(m)\left|\sum_{a=1}^{p-1} e\left(\frac{m a^{4}+a}{p}\right)\right|^{2}=(1+\bar{\psi}(-1))(\tau(\psi)-\sqrt{p} \tau(\bar{\psi}))
$$

Combining (3), (4), and (8) and noting the orthogonality properties of characters $\bmod p$, we have the identity

$$
\begin{aligned}
& \sum_{m=1}^{p-1} \psi(m)\left|\sum_{a=0}^{p-1} e\left(\frac{m a^{4}+a}{p}\right)\right|^{2} \\
& \quad=\sum_{m=1}^{p-1} \psi(m)\left(\sum_{a=1}^{p-1} e\left(\frac{m a^{4}+a}{p}\right)+\sum_{a=1}^{p-1} e\left(\frac{-m a^{4}-a}{p}\right)+\left|\sum_{a=1}^{p-1} e\left(\frac{m a^{4}+a}{p}\right)\right|^{2}\right) \\
& \quad=-(1+\bar{\psi}(-1)) \sqrt{p} \tau(\bar{\psi}) .
\end{aligned}
$$

This proves Lemma 1.

Lemma 2 Let $p$ be an odd prime with $p \equiv 1 \bmod 4$. Then, for the Legendre symbol $\chi_{2} \bmod p$, we have the identity

$$
\sum_{m=1}^{p-1} \chi_{2}(m)\left|\sum_{a=0}^{p-1} e\left(\frac{m a^{4}+a}{p}\right)\right|^{2}=-\psi(-1)\left(\tau^{2}(\psi)+\tau^{2}(\bar{\psi})\right) .
$$

Proof First, from (2) and the method of proving Lemma 1, we have

$$
\begin{aligned}
& \sum_{m=1}^{p-1} \chi_{2}(m)\left|\sum_{a=0}^{p-1} e\left(\frac{m a^{4}+a}{p}\right)\right|^{2} \\
& =\sum_{a=1}^{p-1} \sum_{m=1}^{p-1} \chi_{2}(m) e\left(\frac{m a^{4}+a}{p}\right) \\
& +\sum_{a=1}^{p-1} \sum_{m=1}^{p-1} \chi_{2}(m) e\left(\frac{-m a^{4}-a}{p}\right)+\sum_{m=1}^{p-1} \chi_{2}(m)\left|\sum_{a=1}^{p-1} e\left(\frac{m a^{4}+a}{p}\right)\right|^{2} \\
& =-2 \sqrt{p}+\sum_{m=1}^{p-1} \chi_{2}(m)\left|\sum_{a=1}^{p-1} e\left(\frac{m a^{4}+a}{p}\right)\right|^{2} ; \\
& \sum_{m=1}^{p-1} \chi_{2}(m)\left|\sum_{a=1}^{p-1} e\left(\frac{m a^{4}+a}{p}\right)\right|^{2} \\
& =\sum_{a=1}^{p-1} \sum_{b=1}^{p-1} \sum_{m=1}^{p-1} \chi_{2}(m) e\left(\frac{m b^{4}\left(a^{4}-1\right)+b(a-1)}{p}\right) \\
& =\sqrt{p} \sum_{a=1}^{p-1} \chi_{2}\left(a^{4}-1\right) \sum_{b=1}^{p-1} e\left(\frac{b(a-1)}{p}\right)=-\sqrt{p} \sum_{a=1}^{p-1} \chi_{2}\left(a^{4}-1\right) \\
& =-\sqrt{p} \sum_{a=1}^{p-1} \chi_{2}(a-1)\left(1+\psi(a)+\chi_{2}(a)+\bar{\psi}(a)\right)
\end{aligned}
$$




$$
\begin{aligned}
& =2 \sqrt{p}-\sqrt{p} \sum_{a=1}^{p-1} \chi_{2}(a-1) \psi(a)-\sqrt{p} \sum_{a=1}^{p-1} \chi_{2}(a-1) \bar{\psi}(a) \\
& =2 \sqrt{p}-\psi(-1)\left(\tau^{2}(\psi)+\tau^{2}(\bar{\psi})\right) .
\end{aligned}
$$

Combining (9) and (10), we can deduce the identity

$$
\sum_{m=1}^{p-1} \chi_{2}(m)\left|\sum_{a=0}^{p-1} e\left(\frac{m a^{4}+a}{p}\right)\right|^{2}=-\psi(-1)\left(\tau^{2}(\psi)+\tau^{2}(\bar{\psi})\right) .
$$

This proves Lemma 2.

Lemma 3 Let $p$ be an odd prime with $p \equiv 1 \bmod 4, \psi$ be any fourth-order character $\bmod p$. Then we have the identity

$$
\tau^{2}(\psi)+\tau^{2}(\bar{\psi})=\sqrt{p} \cdot \sum_{a=1}^{p-1}\left(\frac{a+\bar{a}}{p}\right)=2 \sqrt{p} \cdot \alpha .
$$

Proof See Lemma 2.2 in [3].

Lemma 4 Let $p$ be an odd prime with $p \equiv 1 \bmod 4$. Then we have the identity

$$
\sum_{m=1}^{p-1}\left|\sum_{a=0}^{p-1} e\left(\frac{m a^{4}+a}{p}\right)\right|^{2}=p(p-3) .
$$

Proof Since the congruence equation $x^{4} \equiv 1 \bmod p$ has four different solutions in a reduced residue system $\bmod p$, so from (2) we have

$$
\begin{aligned}
\sum_{m=1}^{p-1}\left|\sum_{a=0}^{p-1} e\left(\frac{m a^{4}+a}{p}\right)\right|^{2} & \\
= & \sum_{m=0}^{p-1}\left|\sum_{a=0}^{p-1} e\left(\frac{m a^{4}+a}{p}\right)\right|^{2} \\
= & \sum_{m=0}^{p-1}\left(1+\sum_{a=1}^{p-1} e\left(\frac{m a^{4}+a}{p}\right)+\sum_{a=1}^{p-1} e\left(\frac{-m a^{4}-a}{p}\right)+\left|\sum_{a=1}^{p-1} e\left(\frac{m a^{4}+a}{p}\right)\right|^{2}\right) \\
= & p+\sum_{a=1}^{p-1} \sum_{m=0}^{p-1} e\left(\frac{m a^{4}+a}{p}\right)+\sum_{a=1}^{p-1} \sum_{m=0}^{p-1} e\left(\frac{-m a^{4}-a}{p}\right) \\
& +\sum_{a=1}^{p-1} \sum_{b=1}^{p-1} \sum_{m=0}^{p-1} e\left(\frac{m b^{4}\left(a^{4}-1\right)+b(a-1)}{p}\right) \\
= & p+p(p-1)+\sum_{a=2}^{p-1} \sum_{b=1}^{p-1} \sum_{m=0}^{p-1} e\left(\frac{m b^{4}\left(a^{4}-1\right)+b(a-1)}{p}\right) \\
= & p+p(p-1)-3 p=p(p-3) .
\end{aligned}
$$

This proves Lemma 4. 


\section{Proofs of the theorems}

Now we complete the proofs of our theorems. First we prove Theorem 1. For convenience, we let

$$
B(m)=\sum_{a=0}^{p-1} e\left(\frac{m a^{4}}{p}\right) .
$$

Then, for any integer $m$ with $(m, p)=1$, from (2) and the properties of the fourth-order character $\psi$ mod $p$, we have

$$
\begin{aligned}
B(m)= & 1+\sum_{a=1}^{p-1}\left(1+\psi(a)+\chi_{2}(a)+\bar{\psi}(a)\right) e\left(\frac{m a}{p}\right) \\
= & \sum_{a=0}^{p-1} e\left(\frac{m a}{p}\right)+\sum_{a=1}^{p-1} \psi(a) e\left(\frac{m a}{p}\right) \\
& +\sum_{a=1}^{p-1} \chi_{2}(a) e\left(\frac{m a}{p}\right)+\sum_{a=1}^{p-1} \bar{\psi}(a) e\left(\frac{m a}{p}\right) \\
= & \chi_{2}(m) \sqrt{p}+\bar{\psi}(m) \tau(\psi)+\psi(m) \tau(\bar{\psi}) .
\end{aligned}
$$

If $p=8 r+5$, then $\psi(-1)=-1$. In this case, from Lemma 1 we have the identity

$$
\sum_{m=1}^{p-1} \psi(m)\left|\sum_{a=0}^{p-1} e\left(\frac{m a^{4}+a}{p}\right)\right|^{2}=\sum_{m=1}^{p-1} \bar{\psi}(m)\left|\sum_{a=0}^{p-1} e\left(\frac{m a^{4}+a}{p}\right)\right|^{2}=0 .
$$

It is clear that from Lemma 4 we have

$$
M_{0}(p)=\sum_{m=1}^{p-1}\left|\sum_{a=0}^{p-1} e\left(\frac{m a^{4}+a}{p}\right)\right|^{2}=p(p-3) .
$$

From (12), Lemma 2, and Lemma 3, we have

$$
\begin{aligned}
M_{1}(p) & =\sum_{m=1}^{p-1} B(m)\left|\sum_{a=0}^{p-1} e\left(\frac{m a^{4}+a}{p}\right)\right|^{2} \\
& =\sum_{m=1}^{p-1}\left(\chi_{2}(m) \sqrt{p}+\bar{\psi}(m) \tau(\psi)+\psi(m) \tau(\bar{\psi})\right)\left|\sum_{a=0}^{p-1} e\left(\frac{m a^{4}+a}{p}\right)\right|^{2} \\
& =\sqrt{p}\left(\tau^{2}(\psi)+\tau^{2}(\bar{\psi})\right)=2 p \alpha .
\end{aligned}
$$

Similarly, noting that $\tau(\psi) \tau(\bar{\psi})=-p$, from (12) and Lemma 4 we also have

$$
\begin{aligned}
M_{2}(p) & =\sum_{m=1}^{p-1} B^{2}(m)\left|\sum_{a=0}^{p-1} e\left(\frac{m a^{4}+a}{p}\right)\right|^{2} \\
& =\sum_{m=1}^{p-1}\left(\chi_{2}(m) \sqrt{p}+\bar{\psi}(m) \tau(\psi)+\psi(m) \tau(\bar{\psi})\right)^{2}\left|\sum_{a=0}^{p-1} e\left(\frac{m a^{4}+a}{p}\right)\right|^{2}
\end{aligned}
$$




$$
\begin{aligned}
= & p^{2}(p-3)+\left(\tau^{2}(\psi)+\tau^{2}(\bar{\psi})\right) \sum_{m=1}^{p-1} \chi_{2}(m)\left|\sum_{a=0}^{p-1} e\left(\frac{m a^{4}+a}{p}\right)\right|^{2} \\
& +2 \tau(\psi) \tau(\bar{\psi}) \sum_{m=1}^{p-1}\left|\sum_{a=0}^{p-1} e\left(\frac{m a^{4}+a}{p}\right)\right|^{2} \\
= & p^{2}(p-3)+\left(\tau^{2}(\psi)+\tau^{2}(\bar{\psi})\right)^{2}-2 p^{2}(p-3) \\
= & -p\left(p^{2}-3 p-4 \alpha^{2}\right) ; \\
M_{3}(p)= & \sum_{m=1}^{p-1} B^{3}(m)\left|\sum_{a=0}^{p-1} e\left(\frac{m a^{4}+a}{p}\right)\right|^{2} \\
= & \sum_{m=1}^{p-1}\left(\chi_{2}(m) \sqrt{p}+\bar{\psi}(m) \tau(\psi)+\psi(m) \tau(\bar{\psi})\right)^{3}\left|\sum_{a=0}^{p-1} e\left(\frac{m a^{4}+a}{p}\right)\right|^{2} \\
& +3 \sqrt{p}\left(\tau^{2}(\psi)+\tau^{2}(\bar{\psi})\right) \sum_{m=1}^{p-1}\left|\sum_{a=0}^{p-1} e\left(\frac{m a^{4}+a}{p}\right)\right|^{2} \\
= & p^{\frac{3}{2}} \sum_{m=1}^{p-1} \chi_{2}(m)\left|\sum_{a=0}^{p-1} e\left(\frac{m a^{4}+a}{p}\right)\right|^{2}-6 p^{\frac{3}{2}} \sum_{m=1}^{\chi_{2}(m)}\left|\sum_{a=0}^{p-1} e\left(\frac{m a^{4}+a}{p}\right)\right|^{2} \\
= & 2 p^{2} \alpha(3 p-14) .
\end{aligned}
$$

From [1] (see Lemma 3) we have

$$
B^{4}(m)=-2 p B^{2}(m)+8 p \alpha B(m)-9 p^{2}+4 p \alpha^{2} .
$$

So, if $k \geq 4$, then we have

$$
\begin{aligned}
M_{k}(p) & =\sum_{m=1}^{p-1} B^{k}(m)\left|\sum_{a=0}^{p-1} e\left(\frac{m a^{4}+a}{p}\right)\right|^{2} \\
& =\sum_{m=1}^{p-1} B^{k-4}(m) B^{4}(m)\left|\sum_{a=0}^{p-1} e\left(\frac{m a^{4}+a}{p}\right)\right|^{2} \\
& =\sum_{m=1}^{p-1} B^{k-4}(m)\left(-2 p B^{2}(m)+8 p \alpha B(m)-9 p^{2}+4 p \alpha^{2}\right)\left|\sum_{a=0}^{p-1} e\left(\frac{m a^{4}+a}{p}\right)\right|^{2} \\
& =-2 p M_{k-2}(p)+8 p \alpha M_{k-3}(p)-p\left(9 p-4 \alpha^{2}\right) M_{k-4}(p) .
\end{aligned}
$$

Combining (13)-(16) and (18), we immediately complete the proof of Theorem 1.

Now we prove Theorem 2. If $p=8 k+1$, then note that $\psi(-1)=1$, from Lemma 4 we have

$$
M_{0}(p)=\sum_{m=1}^{p-1}\left|\sum_{a=0}^{p-1} e\left(\frac{m a^{4}+a}{p}\right)\right|^{2}=p(p-3)
$$


From (11), Lemma 1, Lemma 2, and Lemma 3, we have

$$
\begin{aligned}
M_{1}(p) & =\sum_{m=1}^{p-1} B(m)\left|\sum_{a=0}^{p-1} e\left(\frac{m a^{4}+a}{p}\right)\right|^{2} \\
& =\sum_{m=1}^{p-1}\left(\chi_{2}(m) \sqrt{p}+\bar{\psi}(m) \tau(\psi)+\psi(m) \tau(\bar{\psi})\right)\left|\sum_{a=0}^{p-1} e\left(\frac{m a^{4}+a}{p}\right)\right|^{2} \\
& =-3 \sqrt{p}\left(\tau^{2}(\psi)+\tau^{2}(\bar{\psi})\right)=-6 p \alpha
\end{aligned}
$$

Applying Lemma 1, Lemma 2, and Lemma 3, we also have

$$
\begin{aligned}
M_{2}(p)= & \sum_{m=1}^{p-1} B^{2}(m)\left|\sum_{a=0}^{p-1} e\left(\frac{m a^{4}+a}{p}\right)\right|^{2} \\
= & \sum_{m=1}^{p-1}\left(\chi_{2}(m) \sqrt{p}+\bar{\psi}(m) \tau(\psi)+\psi(m) \tau(\bar{\psi})\right)^{2}\left|\sum_{a=0}^{p-1} e\left(\frac{m a^{4}+a}{p}\right)\right|^{2} \\
= & p^{2}(p-3)-\left(\tau^{2}(\psi)+\tau^{2}(\bar{\psi})\right)^{2}-8 p^{2}+2 p^{2}(p-3) \\
= & p\left(3 p^{2}-17 p-4 \alpha^{2}\right) ; \\
M_{3}(p)= & \sum_{m=1}^{p-1} B^{3}(m)\left|\sum_{a=0}^{p-1} e\left(\frac{m a^{4}+a}{p}\right)\right|^{2} \\
= & \sum_{m=1}^{p-1}\left(\chi_{2}(m) \sqrt{p}+\bar{\psi}(m) \tau(\psi)+\psi(m) \tau(\bar{\psi})\right)^{3}\left|\sum_{a=0}^{p-1} e\left(\frac{m a^{4}+a}{p}\right)\right|^{2} \\
= & -p^{\frac{3}{2}}\left(\tau^{2}(\psi)+\tau^{2}(\bar{\psi})\right)-2 p^{\frac{3}{2}}\left(\tau^{2}(\psi)+\tau^{2}(\bar{\psi})\right)-6 p^{\frac{3}{2}}\left(\tau^{2}(\psi)+\tau^{2}(\bar{\psi})\right) \\
& +3 p^{\frac{3}{2}}\left(\tau^{2}(\psi)+\tau^{2}(\bar{\psi})\right)(p-3)-6 p^{\frac{3}{2}}\left(\tau^{2}(\psi)+\tau^{2}(\bar{\psi})\right) \\
= & 6 p^{2} \alpha(p-8) .
\end{aligned}
$$

From [1] (see Lemma 3) we have

$$
B^{4}(m)=6 p B^{2}(m)+8 p \alpha B(m)-p^{2}+4 p \alpha^{2} .
$$

So if $k \geq 4$, then we have the fourth-order linear recurrence formula

$$
\begin{aligned}
M_{k}(p) & =\sum_{m=1}^{p-1} B^{k}(m)\left|\sum_{a=0}^{p-1} e\left(\frac{m a^{4}+a}{p}\right)\right|^{2} \\
& =\sum_{m=1}^{p-1} B^{k-4}(m) B^{4}(m)\left|\sum_{a=0}^{p-1} e\left(\frac{m a^{4}+a}{p}\right)\right|^{2} \\
& =\sum_{m=1}^{p-1} B^{k-4}(m)\left(6 p B^{2}(m)+8 p \alpha B(m)-p^{2}+4 p \alpha^{2}\right)\left|\sum_{a=0}^{p-1} e\left(\frac{m a^{4}+a}{p}\right)\right|^{2} \\
& =6 p M_{k-2}(p)+8 p \alpha M_{k-3}(p)-p\left(p-4 \alpha^{2}\right) M_{k-4}(p) .
\end{aligned}
$$

Now Theorem 2 follows from (19)-(22) and (24). 
If $p \equiv 5 \bmod 8$, then note that $\overline{\tau(\psi)}=-\tau(\bar{\psi})$, from (11) we have

$$
\overline{B(m)}=\chi_{2}(m) \sqrt{p}-\bar{\psi}(m) \tau(\psi)-\psi(m) \tau(\bar{\psi}) .
$$

Thus, from (25) and $\tau(\psi) \tau(\bar{\psi})=-p$, we have

$$
|B(m)|^{2}=p-(\bar{\psi}(m) \tau(\psi)+\psi(m) \tau(\bar{\psi}))^{2}=3 p-\chi_{2}(m)\left(\tau^{2}(\psi)+\tau^{2}(\bar{\psi})\right) .
$$

Applying (26) and Lemma 2, we may immediately deduce Corollary 1.

If $p \equiv 1 \bmod 8$, then note that $B(m)$ is a real number. So Corollary 2 and Corollary 3 follow from Theorem 2 .

This completes the proofs of all our results.

\section{Acknowledgements}

The author would like to thank the referees for their very helpful and detailed comments, which have significantly improved the presentation of this paper.

\section{Funding}

The research was supported by the National Natural Science Foundation of China (Grant No. 11771351; 11501452), the Natural Science Basic Research Plan in Shaanxi Province (Grant No. 2018JQ1093), University's Scientific Research Project (Grant No. 2018KY0208) and the Doctoral Scientific Research Project of Xi'an Aeronautical University.

\section{Competing interests}

The author declares that there is no conflict of interests regarding the publication of this paper.

\section{Authors' contributions}

The author read and approved the final manuscript.

\section{Publisher's Note}

Springer Nature remains neutral with regard to jurisdictional claims in published maps and institutional affiliations.

Received: 1 May 2018 Accepted: 30 May 2018 Published online: 11 July 2018

\section{References}

1. Shen, S.M., Zhang, W.P.: On the quartic Gauss sums and their recurrence property. Adv. Differ. Equ. 2017, Article ID 43 (2017)

2. Li, X.X., Hu, J.Y.: The hybrid power mean quartic Gauss sums and Kloosterman sums. Open Math. 15, 151-156 (2017)

3. Chen, Z.Y., Zhang, W.P.: On the fourth-order linear recurrence formula related to classical Gauss sums. Open Math. 15, $1251-1255(2017)$

4. Zhang, W.P., Liu, H.N.: On the general Gauss sums and their fourth power mean. Osaka J. Math. 42, 189-199 (2005)

5. Han, D.: A hybrid mean value involving two-term exponential sums and polynomial character sums. Czechoslov. Math. J. 64, 53-62 (2014)

6. Zhang, W.P., Han, D.: On the sixth power mean of the two-term exponential sums. J. Number Theory 136, 403-413 (2014)

7. Cochrane, T.: Exponential sums modulo prime powers. Acta Arith. 101, 131-149 (2002)

8. Cochrane, T., Pinner, C.: Using Stepanov's method for exponential sums involving rational functions. J. Number Theory 116, 270-292 (2006)

9. Ren, G.L.: On the fourth power mean of one kind special quadratic Gauss sums. J. Northwest Univ. Nat. Sci. 46(3), 321-324 (2016)

10. Zhang, H., Zhang, W.P.: The fourth power mean of two-term exponential sums and its application. Math. Rep. 19, 75-83 (2017)

11. Zhang, W.P., Hu, J.Y.: The number of solutions of the diagonal cubic congruence equation mod $p$. Math. Rep. 20, 73-80 (2018)

12. Zhang, W.P., Li, H.L.: Elementary Number Theory. Shaanxi Normal University Press, Xi'an (2008)

13. Apostol, T.M.: Introduction to Analytic Number Theory. Springer, New York (1976) 